\title{
Randomized Control Trial to Assess the Efficacy and Safety of NRL/2019/5PNW with Micronutrient Fortification to Promote Health and Well-being in Woman in Fertile Age
}

\author{
Priya Khare ${ }^{1, *}$, Omkar Kulkarni ${ }^{1}$, Anuj Agrawal ${ }^{1}$, Gayatri Ganu ${ }^{2}$, Medha Joshi $^{3}$, Manjit Sisode ${ }^{4}$ \\ 'Netsurf Research Labs Pvt. Ltd, Baner, Pune, Maharashtra, INDIA. \\ ${ }^{2}$ Clinical Research Unit, Mprex Healthcare, Nisarg Plaza, Bhumkar Nagar, Wakad, Pune, Maharashtra, INDIA. \\ ${ }^{3}$ Pune District Education Association, Pune College of Ayurveda and Sterling Hospital Research Center, Pune, Maharashtra, INDIA. \\ ${ }^{4}$ Janseva Multispecialty Hospital, Old Agra Rd, Deopur, Dhule, Maharashtra, INDIA.
}

\begin{abstract}
Background:Women appear with gender specific nutritional requirements that may affect their well-being. The nutritional requirements are there for women in rural, suburban and urban regions altogether. Objective: Netsurf Communications Pvt. Ltd. has developed the NRL/2019/5PNW for the women to help manage their health and wellness. The current research is designed to assess safety and efficacy of the test product. Methods and Materials: The trial is randomized, controlled prospective clinical trial. In the present study, 110 subjects were screened. Total 100 subjects were considered evaluable cases at the end of the study 1:1 in test and Marketed product treated group. Results: Test product was better in activity than marketed product in improving energy levels of the subjects throughout the day, elevating mood, improving quality of sleep and reducing stress. Improved glycemic control and reduction in total cholesterol levels
\end{abstract}

were significantly reduced in test group which has preventive potential of metabolic diseases. Less events of recurrent UTI/ RTI in test treated group suggestive of improvement in immune status of females. Conclusion: NRL/2019/5PNW is safe and effective in promoting health and wellbeing in females in fertile age.

Key words: Curcumin, Energy level, Protein supplement, Soya, Whey.

Correspondence

Dr. Omkar Kulkarni

Senior Manager, R\&D (Healthcare), Netsurf Research Labs Pvt. Ltd, Baner, Pune-411045, Maharashtra, INDIA.

Phone no: +91 8554912644

Email: omkar.kulkarni@nrl.net.in

DOI: 10.5330/ijpi.2020.4.101

\section{INTRODUCTION}

A woman's nutritional and fitness requirements are different than men as there are indeed some special requirements of women to lose fat, gain muscle and look lean. Protein maintain cellular structures, hair, bones and connective tissue. Proteins account for digestive enzymes, immune system and muscle strength. Immune system functionality can be improvised by proper protein supplements. Bone health and density keeps bones strong and minimize the density loss that could be anticaking. Proteins help hair and nails looking healthy and strong thus improving wellness quotient. ${ }^{1}$

Whey protein, soya protein together with vegetable proteins like rice, pea is a better blend in terms of presence of amino acids present and digestibility and thus assimilation. Plant-based proteins tend to be lower in calories and fat than animal proteins and higher in fiber and essential nutrients. Plant-based proteins made with peas can promote muscle thickness gains. In turn vegetable proteins help reducing hunger cravings, are easy at digestions and leads to less gastric distress than animal based source. ${ }^{2}$

Women have gender specific nutritional requirements that may affect wellbeing and there is great need to address the same. The requirements are there in rural, sub-urban and urban regions altogether. Anatomy and physiological stages of females and role of sex hormones throughout lifetime present nutritional challenges requiring special attention. ${ }^{3}$ Physical demands on females during fertile age affect nutrition, appetite and health status. Cultural and social factors resulting in stressing gender-specific roles, body shape and weight put woman on risk of eating disorders and seeking unhealthy methods to lose weight. Neural and hormonal factors associate to mood swings and perception to stress in woman life. ${ }^{4}$ Women present many nutrition-related concerns from weight maintenance to bone health. By taking in to consideration the special need of good protein for women, the Netsurf Communications Pvt. Ltd. has designed and developed the NRL/2019/5PNW for the women to help to manage health and wellness. The present study aims and clinical validation of safety and efficacy of woman protein supplement in their fertile age. ${ }^{5}$

\section{MATERIALS AND METHODS}

The study was initiated after Ethics Committee approval and subsequent registration of study on CTRI website (CTRI/2019/10/021717 [Registered on: 18/10/2019]). The study was conducted as per Good Clinical Practices guidelines. Patients were enrolled in the study only after registration of study on CTRI website.

The primary objectives of the study were to evaluate efficacy of NRL/2019/5PNW and marketed product in fertile women by assessingdaily energy levels, menstrual regulation, changes in biochemical parameters, immunity via number of events of recurrent UTI/RTI etc., changes in hormonal profile.

The secondary objectives of the study were to assess- quality of life (Physical, mental and social) General Health Questionnaire-28 (GHQ28 ), changes in mood behavior, sleep quality questionnaire, perceived stress questionnaire, safety assessment. 


\section{Inclusion Criteria}

Fertile Women Aged 18 - 50 years with BMI in the range of $20-26 \mathrm{Kg} / \mathrm{m}^{2}$ were incorporated. Subjects having good physical condition and sound mental status and in the judgment of the principal investigator, able to comply with protocol requirements were included. Subjects willing to sign written informed consent and willing to abstain from use of additional vitamin or mineral supplements, nutritional supplements and or medical foods if applicable were recruited in the trial.

\section{Exclusion Criteria}

Subjects with medical history of any major illness such as cancer, heart disease, COPD, Asthma etc. in the past were excluded. Subjects with history of any acute or chronic illness that may affect the patient's participation in the study were excluded. Subjects on prescription medications and/or nonprescription medications for weight loss were not considered in the study. Pregnant and/or breast feeding subjects, not willing to participate in study, subjects with history of hysterectomy, IUI and IVF Subjects with PCOS and or on any hormone replacement therapy were excluded. NRL/2019/5PNW powder is approved by FSSAI. It contains protein blend (whey protein concentrate, soy protein isolate, pea protein isolate, rice protein isolate), milk solids DHA (omega 3-Algal source), curcumin, shatavari extract, multivitamins and multiminerals.

As per computer generated randomization list, subject were either be randomized to test or marketed product group in 1:1 ratio. Dose of 10 gm. in the morning with $150 \mathrm{ml}$ of milk for 90 days were administered. Visit schedule were on baseline visit, day 30, 60 and 90 .

\section{Study procedure}

Fertile female subjects of age between 18 to 50 years of age (both inclusive) attending outpatient department of study site(s) were screened for eligibility criteria. On screening visit, a written informed consent was obtained from subjects for their participation in the study. Subject's demographic details and clinical examination was recorded. Subject's medical, surgical and treatment history was taken. Subject's current medication if any was noted in the case record from (CRF). The subject was considered for further evaluation as per the inclusion and exclusion criteria. All biochemical parameters were recorded at screening visit. During screening visit and the entire study duration subjects were advised to refrain from antioxidant agents, vitamins, anti-inflammatory drugs, hormones, Nutraceutical, Ayurvedic, Siddha, Unani, herbal / homeopathic medicines. Subjects were checked for occurrence of any adverse event during screening period. Subject's daily energy levels, changes in menstrual regulation, anthropometric analysis and immunity events were assessed. Subjects were assessed for follow up visits after every month up to 3 months. On last visit the biochemical tests were repeated. All clinical and questionnaires were assessed for all visits. All the subjects were closely monitored for any adverse event.

Table 1: Assessment of Mood Disorder Questionnaire (MDQ) Score between the groups.

\begin{tabular}{ccc}
\hline $\begin{array}{c}\text { Duration } \\
\text { (Days) }\end{array}$ & \multicolumn{2}{c}{ Mean Mood Disorder Questionnaire (Mean \pm SD) } \\
\cline { 2 - 3 } Baseline & Test $(\mathbf{N}=\mathbf{5 0})$ & Marketed Product $(\mathbf{N}=\mathbf{5 0})$ \\
\hline 30 & $2.04 \pm 0.51$ & $1.98 \pm 0.63$ \\
60 & $2.04 \pm 0.47$ & $2.00 \pm 0.48$ \\
90 & $2.41 \pm 0.54^{* *}$ & $2.02 \pm 0.40$ \\
\hline
\end{tabular}

By ANOVA Test: $P>0.05$ Not Significant; significant $p<0.01^{*}, p<0.001^{* *}$

\section{Sample size consideration}

Sample size calculation is derived taking considerations of primary and secondary outcomes by a qualified statistician. The software used for calculation of sample size is SPSS version 10.0

\section{RESULTS}

\section{Demographic details}

In the present study, 110 subjects were screened. Out of 110 subjects, 3 subjects were screen failure and 7 lost to follow up in the study. 100 subjects were considered evaluable cases at the end of the study 50 in test and 50 in Marketed product treated group.

Out of 100 completed subjects, the mean age of Test group subjects in were $35.67 \pm 8.01$ years and the mean age of Marketed product group subjects was $34.38 \pm 7.36$ years. If compared between the groups, the difference was statistically insignificant.

\section{Assessment of mood disorder questionnaire (MDQ) score}

There was significant $(p<0.001)$ increase in MDQ score in Test product compared to Marketed product treated group after day 60 and 90 . The results are given in Table 1.

\section{Assessment of Pittsburgh sleep quality index (PSQI):}

There was significant decrease $(p<0.001)$ between PSQI in Test product and Marketed product treated group after day 60 and 90 . The results are given in Table 2.

\section{Assessment of perceived stress score (PSS)}

There was significant decrease $(p<0.001)$ between PSS in Test product and Marketed product treated group after day 60 and 90 . The results are given in Table 3.

\section{Assessment of general heath questionnaire-28 (GHQ-28)} score

At 30, 60 and 90 days there was significant $(p<0.001)$ increase in GHQ28 score of Test product compared to Marketed product treated group. The results are given in Table 4 .

\section{Assessment of energy audit questionnaire}

The frequency distribution of energy events depicted difference in "Very High" and "High" score is increased significantly in test treated group than marketed product treated group. The results are given in Table 5.

\section{Assessment of anthropometric parameters}

There were significant $(p<0.05)$ reduction in fat $\%$ at day 60 and 90 , in test group when compared to marketed product treated group. There

\section{Table 2: Assessment of Pittsburgh Sleep Quality Index (PSQI) between} the groups.

\begin{tabular}{ccc}
\hline \multirow{2}{*}{$\begin{array}{c}\text { Duration } \\
\text { (Days) }\end{array}$} & \multicolumn{2}{c}{ Mean Pittsburgh Sleep Quality Index(Mean \pm SD) } \\
\cline { 2 - 3 } Baseline & Test $(\mathrm{N}=50)$ & Marketed Product $(\mathrm{N}=50)$ \\
30 & $5.52 \pm 2.57$ & $5.48 \pm 2.43$ \\
60 & $4.28 \pm 2.40$ & $5.43 \pm 2.37$ \\
90 & $2.48 \pm 1.47^{* *}$ & $4.85 \pm 2.44$ \\
\hline
\end{tabular}

By ANOVA multiple comparison Test: $P>0.05$ Not Significant; significant $p<$ $0.01^{\star}, p<0.001^{* *}$ 
were significant $(p<0.05)$ reduction in visceral fat $\%$ at day 60 and 90 , in test group when compared to marketed product treated group. There was small increase in body weight in both groups after treatment but the comparison was not significant.

\section{Assessment of iron profile}

There was significant $(p<0.05)$ increase in serum iron in test group when day 90 results $(73.45 \pm 22.51)$ were compared to baseline results $(69.14 \pm 27.02)$ of same group. There was significant $(p<0.05)$ increase in ferritin levels at day 90 between test and marketed product treated group. The mean values of ferritin were 51.34 \pm 5.98 and 45.27 in test and marketed product treated group respectively.

\section{Assessment of hormonal profile}

The hormonal ranges were normal in all subjects and there was no significant changes in serum levels of FSH, LH, PRL and testosterone after treatment with test and marketed product treated group.

\section{Assessment of immunity events by recurrent RTI/ UTI}

Total of 15 subjects of test group reported average of 15 events of RTI/ UTI prior to last month of trial commencement. Total 10 subjects (20\%) reported recurrent episodes of either RTI/UTI during three months period. Total 12 subjects of marketed product treated group reported average of at least 13 events of RTI/ UTI prior to last month of trial commencement. In marketed product group, 15 (30) subjects reported 18 events of recurrent RTI/ UTI during three months period and found to be completely resolved in two to three days.

\section{Assessment of menstrual profile}

Total 5 subjects out of 50 ie $10 \%$ of test group subjects' experiences profuse blood flow at baseline of which only 2 subjects were reporting profuse blood flow after 3 months of treatment with test product. In case of marketed product, 4 out of 50 of marketed product group subject's

Table 3: Assessment of Perceived Stress Score (PSS) between the groups.

\begin{tabular}{ccc}
\hline \multirow{2}{*}{$\begin{array}{c}\text { Duration } \\
\text { (Days) }\end{array}$} & Mean Perceived stress questionnaire(Mean $\pm \mathrm{SD})$ \\
\cline { 2 - 3 } Baseline & Test $(\mathrm{N}=50)$ & Marketed Product(N = 51) \\
30 & $17.50 \pm 3.34$ & $18.18 \pm 3.18$ \\
60 & $17.17 \pm 2.51$ & $17.52 \pm 3.05$ \\
90 & $11.93 \pm 3.43^{* *}$ & $17.52 \pm 3.14$ \\
\hline
\end{tabular}

By ANOVA multiple comparison Test: $P>0.05$ Not Significant; significant $p<$ $0.01^{*}, p<0.001^{* *}$

Table 4: Assessment of General Heath Questionnaire-28 (GHQ-28) Score between the groups.

\begin{tabular}{ccc}
\hline \multirow{2}{*}{$\begin{array}{c}\text { Duration } \\
\text { (Days) }\end{array}$} & \multicolumn{2}{c}{ Mean General Heath Questionnaire-28(Mean \pm SD) } \\
\cline { 2 - 3 } Baseline & Test $(\mathrm{N}=50)$ & Marketed Product $(\mathrm{N}=50)$ \\
\hline 30 & $52.70 \pm 9.62$ & $47.61 \pm 5.38$ \\
60 & $71.39 \pm 6.04^{* * *}$ & $43.43 \pm 5.98$ \\
90 & $78.98 \pm 5.55^{* * *}$ & $58.82 \pm 9.18$ \\
\hline
\end{tabular}

By ANOVA multiple comparison Test: $P>0.05$ Not Significant; significant $p<0.001^{* *}, p<0.0001^{* * *}$ experienced profuse blood flow at baseline and 5 subjects were reporting profuse blood flow after 3 months of treatment with test product.

\section{Changes in Mean parameters of complete hemogram,} liver, renal, thyroid function test, serum insulin and random blood sugar levels

All the parameters in the complete hemogram, liver, renal, thyroid function test, serum insulin and random blood sugar levels were within normal range and comparable between groups at baseline. After treatment at day 90, mean value of parameters of said parameters did not show any significant change from baseline in test and marketed product treated groups. The difference was statistically insignificant.

\section{Changes in mean parameter of lipid profile}

There was significant $p<0.05$ reduction in total cholesterol of test product treated group at day 90 . The results are given in Table 6.

\section{Changes in mean $\mathrm{HbA} 1 \mathrm{C}$}

After completion of the treatment, there was significant decrease in $\mathrm{HbA}_{1 \mathrm{C}}$ level in test treated group compared to marketed product treated groups. The results are given in Table 7.

\section{Changes in vital parameters}

Pulse, respiratory rate, blood pressure of subjects of both the groups was within normal limits at baseline visit. After completion of the treatment, no significant change in said parameters.

\section{Tolerability of study drugs assessed by physician and subjects}

As per physician and subjects, all the subjects (100\%) from both the groups reported excellent tolerability to given intervention i e test product and marketed product.

\section{Profile of adverse events}

There were 20 (40\%) and 18 (36\%) subjects reported a total of 24 and 26 adverse events during the study period respectively in test and marketed product treated group. These adverse events included fever, menstrual pain, hyperacidity, injury, body ache, urinary irritation, cold, heartburn and headache. These adverse events were resolved completely after rescue medication was given. Study treatment was not stopped during these adverse events. All these adverse events were not related to the study drug.

\section{DISCUSSION}

Women of reproductive age experience many emotional disturbances, of which $20-50 \%$ percent have a broad symptomatology. These emotional distress could be a sign of premenstrual syndrome that could be not distinguished presentation so far. Mood fluctuations are very common and profound symptom in many woman which affects her quality of life. The irritable and depressed moods alters the perception to the stress triggers and develop perceived stress. ${ }^{6}$ In the present study NRL/2019/5PNW found to be significant in improving mood status in females than in marketed product treated group from day 60 . The activity of the NRL/2019/5PNW would be due to improved energy levels due to protein intake and micronutrients supplementation like vitamins and minerals.

Women show up with poorer sleep quality than men and possess risk for insomnia. Disturbed sleep could be attributed to reproductive hormones, stress, depression, aging, societal and family liabilities. ${ }^{7}$ Reduced Pittsburgh Sleep Quality Index indicated that the NRL/2019/5PNW 
Table 5: Assessment of Energy Audit Questionnaire Mean Score between the groups.

\begin{tabular}{ccccccc} 
& \multicolumn{5}{c}{ Mean Energy Audit(Mean $\pm \mathrm{SD}$ ) } \\
\cline { 2 - 7 } Energy Level & \multicolumn{5}{c}{ Dest $(\mathrm{N}=50)$} & \multicolumn{3}{c}{ Marketed Product (N=50) } \\
\cline { 2 - 7 } & Day 30 & Day 60 & Day 90 & Day 30 & Day 60 & Day 90 \\
\hline Very High & $4.00 \pm 3.93$ & $23.35 \pm 11.13$ & $24.46 \pm 7.61$ & $3.91 \pm 4.00$ & $10.73 \pm 8.69$ & $14.02 \pm 5.34$ \\
High & $5.96 \pm 4.85$ & $31.52 \pm 10.15$ & $27.96 \pm 7.26$ & $6.05 \pm 4.45$ & $19.43 \pm 14.81$ & $23.41 \pm 7.73$ \\
Moderate & $35.35 \pm 13.70$ & $27.11 \pm 9.62$ & $23.61 \pm 7.77$ & $34.09 \pm 14.57$ & $37.66 \pm 10.93$ & $32.84 \pm 9.00$ \\
Neutral & $38.39 \pm 15.09$ & $24.61 \pm 9.86$ & $20.78 \pm 6.14$ & $39.11 \pm 15.76$ & $37.82 \pm 14.44$ & $25.70 \pm 9.05$ \\
Low & $15.22 \pm 10.37$ & $7.78 \pm 4.80$ & $2.57 \pm 2.40$ & $15.23 \pm 10.93$ & $8.77 \pm 5.24$ & $2.59 \pm 2.35$ \\
Very low & $19.93 \pm 21.28$ & $4.67 \pm 3.91$ & $0.91 \pm 1.98$ & $20.36 \pm 21.25$ & $5.18 \pm 4.73$ & $0.86 \pm 1.64$ \\
\hline
\end{tabular}

By ANOVA multiple comparison Test: $P>0.05$ Not Significant; significant $p<0.001^{* *}, p<0.0001^{* * *}$

Table 6: Changes in Mean parameters of lipid profile between the groups.

\begin{tabular}{ccccc}
\hline \multirow{2}{*}{ Lipid Profile } & \multicolumn{4}{c}{ Mean(Mean \pm SD) } \\
\cline { 2 - 5 } & \multicolumn{2}{c}{ Test $(\mathrm{N}=50)$} & \multicolumn{2}{c}{ Marketed Product $(\mathrm{N}=50)$} \\
\cline { 2 - 5 } & Baseline & Day 90 & Baseline & Day 90 \\
\hline Total Cholesterol $(\mathrm{mg} / \mathrm{dl})$ & $164.22 \pm 28.42$ & $155.26 \pm 32.62^{*}$ & $166.91 \pm 30.20$ & $169.70 \pm 25.40$ \\
Cholesterol HDL Direct $(\mathrm{mg} / \mathrm{dl})$ & $54.57 \pm 11.69$ & $52.96 \pm 13.54$ & $56.17 \pm 11.92$ & $55.35 \pm 12.52$ \\
Triglycerides $(\mathrm{mg} / \mathrm{dl})$ & $126.84 \pm 59.50$ & $129.53 \pm 58.75$ & $129.70 \pm 38.94$ & $128.80 \pm 45.05$ \\
LDL Cholesterol $(\mathrm{mg} / \mathrm{dl})$ & $103.65 \pm 20.61$ & $101.76 \pm 25.69$ & $108.12 \pm 18.11$ & $106.93 \pm 16.65$ \\
VLDL Cholesterol $(\mathrm{mg} / \mathrm{dl})$ & $25.98 \pm 10.09$ & $27.38 \pm 10.42$ & $26.56 \pm 9.43$ & $28.26 \pm 10.44$ \\
\hline
\end{tabular}

By Student's t Test: $P>0.05$ Not Significant, $P<0.05$ Significant ${ }^{*}$

treated group experiences less disturbed sleep with less mid night awakenings and sleep latency however improved onset of sleep and duration of uninterrupted sleep etc.

One of the most widely used tool for measuring the perception of stress is the Perceived Stress Scale (PSS). This scale evaluates the degree to which situations in one's life are judged as stressful. ${ }^{8}$ At day 60 and 90, NRL/2019/5PNW demonstrated significant reduction in PSS suggestive of lesser appraisal of stress in situations of life by subjects treated with NRL/2019/5PNW. The reduced perception to stress could be due to reduced mood fluctuations, improved sleep and energy levels.

The assessment of the GHQ-9 score depicted that there was improved score at day 30,60 and 90 in NRL/2019/5PNW treated group than marketed product treated group.

Physical health and well-being is feeling of energy and stamina. There was assessment of energy audit questionnaire to assess subjective changes of the energy levels of the subject divided in 4 parts of day i.e. early morning till breakfast, Breakfast to lunch, Lunch to evening snack time and late evening to bedtime. The energy levels were divided into Very high, High, Moderate, Neutral, Low and Very low depending on the expression of the energy at particular time. ${ }^{9}$ The subject reported their reading in terms of score for the day. The results are expressed as mean score and frequency of events in each score title. There was significant increase in shift of individuals experiencing Very High and High energy evidences than Low, Moderate and Neutral energy level in NRL/2019/5PNW treated group than that of marketed product. Energy levels are strongly depend on the muscular strength and vitality together with improved mood status and reduced perceived stress.

Excessive visceral fat is associated with decreased insulin sensitivity which in turn increases risk to cardiovascular disease. In case of woman the visceral fat can induce deposition of reproductive hormone and thus potentiates risk of PCOD etc. ${ }^{10}$ The reduction in visceral fat in
Table 7: Changes in Mean $\mathrm{HbA1C} \%$ between the groups.

\begin{tabular}{ccc|}
\hline \multirow{2}{*}{$\begin{array}{c}\text { Duration } \\
\text { (Days) }\end{array}$} & \multicolumn{2}{c|}{ Mean HbA1c \%(Mean $\pm \mathrm{SD})$} \\
\cline { 2 - 3 } Baseline & Test $(\mathrm{N}=50)$ & Marketed Product $(\mathrm{N}=50)$ \\
90 & $5.17 \pm 0.49$ & $5.15 \pm 0.52$ \\
\hline
\end{tabular}

Student's $t$ test : $P>0.05$ Not Significant, $P<0.05$ Significant $^{*}$

NRL/2019/5PNW treated group can prove beneficial in preventing these complications.

Ferritin is a protein that stores iron. Ferritin are present in hepatocytes and reticuloendothelial cells. The ferritin binds to another substance called transferrin. It combines with ferritin to transport it to where new red blood cells are made. It's not only important to have normal iron levels, but should have enough stored iron. From the data of present study it is demonstrated that NRL/2019/5PNW improved ferritin levels significantly at day 90 than marketed product. In the data obtained in study, serum iron, total iron binding capacity and transferrin \% are increased in both groups magnitude wise but non-significant in comparison. Iron helps to preserve many vital functions in the body, including general energy and focus, gastrointestinal processes, the immune system and the regulation of body temperature. It indicated the ability of NRL/2019/5PNW to improve iron profile in fertile females. This could lead in improved energy, stamina and health status.

As there are positive changes in the energy, stamina, mental state and quality, there are positive changes in immunity status as indicated by events of URI/ RTI in treatment months.

Recent research evidence that curcumin has strong anti-inflammatory and antioxidant activities and modulates inflammatory responses 
which are underlying cause to many diseases. Curcumin possess immunomodulatory effects with multilayer response such as effect on lymphoid cell populations, antigen presentation, humoral and cellmediated immunity and cytokine production. Curcumin present in NRL/2019/5PNW can play good role in modifying immunity of subjects. ${ }^{11} \mathrm{NRL} / 2019 / 5 \mathrm{PNW}$ possess extract of Asparagus racemosus ieshatavari. The roots of shatavari are tonic and diuretic and galactgogue and possess ulcer healing effect probably via strengthening the mucosal resistance or gastro-cytoprotection. A. racemosus is widely used from ancient times in keeping woman health by virtue of antihepatotoxic, immunomodulatory effects, immune adjuvant effect, uterine tonic and general tonic and rejuvenator properties. ${ }^{12}$ Glycemic regulation is thought to be a key in maintaining healthy metabolism and preventing many metabolic diseases like diabetes, cardiovascular diseases. Protein supplements changes all paradigm of metabolism and manages to get good glycemic conditions when person is not no high fat diet. ${ }^{13}$ Soy protein is reported to possess low glycemic index. Plant proteins like rice and pea proteins are reported to alter the glycemic and appetitive responses. ${ }^{14}$ In the present study, though random blood sugar is not significantly reduced, but the $\mathrm{HbA}_{1 \mathrm{C}}$ levels are significantly reduced in NRL/2019/5PNW treated group.

It was evident that soy protein supplementation may effectively reduce serum cholesterol levels and therefore is likely to diminish the risk for cardiovascular disease. ${ }^{15}$ In the present study there was significant $(p<0.05)$ reduction in total cholesterol in NRL/2019/5PNW treated group at day 90 of treatment.

\section{CONCLUSION}

In comparison to marketed product with a protein blend with multivitamin, NRL/2019/5PNW showed marked improvement in stress score, sleep pattern, glycemic control than marketed product. NRL/2019/5PNW produced reduction in cholesterol levels and visceral fat than marketed product. There was no significant post treatment change in biochemical teats in both the groups, suggesting both the drugs were safe in human subjects. Thus NRL/2019/5PNW is safe and effective in promoting health and wellbeing in fertile females.

\section{ACKNOWLEDGEMENT}

Authors wish to acknowledge Netsurf Communications Pvt. Ltd. for funding this project and supporting conducting this study.

\section{CONFLICT OF INTEREST}

There are no conflicts of interest.

\section{REFERENCES}

1. Messina MJ. Legumes and soybeans: Overview of their nutritional profiles and health effects. Am J Clin Nutr. 1999;70(3):439s-50s.

2. Thomson WA. Infant formulas and the use of vegetable protein. J Am Oil Chem' Society. 1979;56(3):386-8.

3. Henderson L, Gregory J, Swan G. The National Diet and Nutrition Survey: Adults aged 19 to 64 years. Vitamin and Mineral Intake and Urinary Analytes. 2003;3:1-8.

4. Francis RM. What do we currently know about nutrition and bone health in relation to United Kingdom public health policy with particular reference to calcium and vitamin D?. Br J Nutr. 2008;99(1):155-9.

5. Burns L, Ashwell M, Berry J, Bolton-Smith C, Cassidy A, Dunnigan M, et al. UK Food Standards Agency Optimal Nutrition Status Workshop: Environmental factors that affect bone health throughout life. Br J Nutr. 2003;89(6):835-40.

6. Bowen R, Bowen A, Baetz M, Wagner J, Pierson R. Mood instability in women with premenstrual syndrome. J Clin Gynecol Obstet. 2011;33(9):927-34.

7. Nowakowski S, Meers J, Heimbach E. Sleep and women's health. Sleep Med Res. 2013;4(1):1.

8. Cohen S, Kamarck T, Mermelstein R. A global measure of perceived stress. J Health Soc Behav. 1983;385-96.

9. Spreitzer GM, GrantT. Helping students manage their energy: Taking their pulse with the energy audit. Journal of Management Education. 2012;36(2):239-63.

10. Bosch TA, Steinberger J, Sinaiko AR, Moran A, Jacobs DR, et al. Identification of sex-specific thresholds for accumulation of visceral adipose tissue in adults. Obesity. 2015;23(2):375-82.

11. Hoie LH, Graubaum HJ, Harde A, Gruenwald J, Wernecke KD. Lipid-lowering effect of 2 dosages of a soy protein supplement in hypercholesterolemia. Adv ther. 2005;22(2):175-86

12. Alok S, Jain SK, Verma A, Kumar M, Mahor A, Sabharwal M. Plant profile, phytochemistry and pharmacology of Asparagus racemosus (Shatavari): A review. Asian Pac J Trop Dis. 2013;3(3):242-51.

13. Stevenson EJ, Allerton DM. The role of whey protein in postprandial glycaemic control. Proceedings of the Nutrition Society. 2018;77(1):42-51.

14. Tan SY, Siow PC, Peh E, Henry CJ. Influence of rice, pea and oat proteins in attenuating glycemic response of sugar-sweetened beverages. Eur J Nutr. 2018;57(8):2795-803.

15. Antony S, Kuttan R, Kuttan G. Immunomodulatory activity of curcumin. Immunol Invest. 1999;28(5-6):291-303.

Article History: Submission Date : 05-09-2020; Revised Date : 11-10-2020; Acceptance Date : 25-10-2020.

Cite this article: Khare P, Kulkarni O, Agrawal A, Ganu G, Joshi M, Sisode M. Randomized Control Trial to Assess the Efficacy and Safety of NRL/2019/5PNW with Micronutrient Fortification to Promote Health and Well-being in Woman in Fertile Age. Int. J. Pharm. Investigation, 2020;10(4):581-5. 